\title{
Impact of convective downdrafts on model simulations: results from aqua-planet integrations
}

\author{
S. Sahany and R. S. Nanjundiah \\ Centre for Atmospheric and Oceanic Sciences, Indian Institute of Science, Bangalore-560012, India \\ Received: 8 August 2007 - Revised: 26 March 2008 - Accepted: 25 April 2008 - Published: 10 July 2008
}

\begin{abstract}
The role of convective scale downdrafts has been examined, using the NCAR-CAM3.0 aqua-planet configuration. We find that, convective downdrafts make the atmosphere more unstable thus increasing the convective available potential energy (CAPE) of the atmosphere. It is noticed that, although the rate of CAPE consumption increases with the incorporation of downdrafts, the generation of CAPE increases with a higher rate. Also, it is noted that there is a reduction in the deep convective rainfall, with the inclusion of downdrafts, which is primarily due to the re-evaporation of precipitation within the downdrafts. There is a large increase in the low cloud fraction and the shortwave cloud forcing with the inclusion of convective scale downdrafts in the cumulus scheme, which along with the evaporation within the downdraft causes cooling in the troposphere.

This is in contrast to previous studies on the impact of downdrafts using single column models. In Zhang and McFarlane (1995), using a single column model, it was shown that with the increase in the strength of the downdrafts, there was a reduction in CAPE. In the present study, using an aquaplanet framework, it is shown that CAPE is actually found to increase when the downdrafts are incorporated into the cumulus scheme, as compared to the case when there are no downdrafts. The rate of CAPE consumption which, is the same as the rate of stabilization of the atmosphere, is found to increase, but the mean CAPE as such is higher compared to the case when there are no downdrafts.
\end{abstract}

Keywords. Meteorology and atmospheric dynamics (Convective processes; Precipitation; Tropical meteorology)

Correspondence to: S. Sahany

(sandeep@caos.iisc.ernet.in)

\section{Introduction}

Aqua-planets are a useful test-bed to study various submodels of the atmosphere. In an aqua-planet while all the atmospheric interactions are present, feedback effects from and to the surface are absent, thus making it a useful testbed. Aqua-planets have been used extensively to study various aspects of tropical climate (Neale and Hoskins, 2000a, b). Neale and Hoskins (2000a) proposed a suite of aqua-planet simulations as a standard test for Atmospheric General Circulation Models (AGCMs). Their suite is intended to provide an experimental protocol which is less complex than the full AGCM but more complex than the single column model. The motivation behind this work is to understand the role of the convective downdrafts on the simulation of mean atmospheric state, rather than on a particular climatic feature, so we preferred to use the aqua-planet configuration. We have carried out the Neale and Hoskins suite of simulations with the standard CAM3.0 which uses the Zhang and McFarlane (1995) cumulus scheme for the parameterization of deep convection.

Zhang and McFarlane (ZM) had carried out extensive studies on the performance of the scheme and its sensitivity to different parameters in a Single Column Model (SCM) framework, as well as in a three-dimensional GCM, using the Canadian Climate Center General Circulation Model (CCC GCM). They clearly showed that, model simulations are sensitive to the strength of the downdraft. Their SCM results show that downdrafts tend to reduce the mean CAPE in the atmosphere. Based on the above results, they stated that strong downdrafts provide a powerful mechanism for stabilizing the atmospheric column. Molinari and Corsetti (1985), using the Kuo scheme, showed that the inclusion of downdrafts increases the rate of stabilization of the atmosphere. The common conclusion which is drawn from the above two GCM works is that downdrafts increase the rate of stabilization of the atmosphere. But the question which is still

Published by Copernicus Publications on behalf of the European Geosciences Union. 


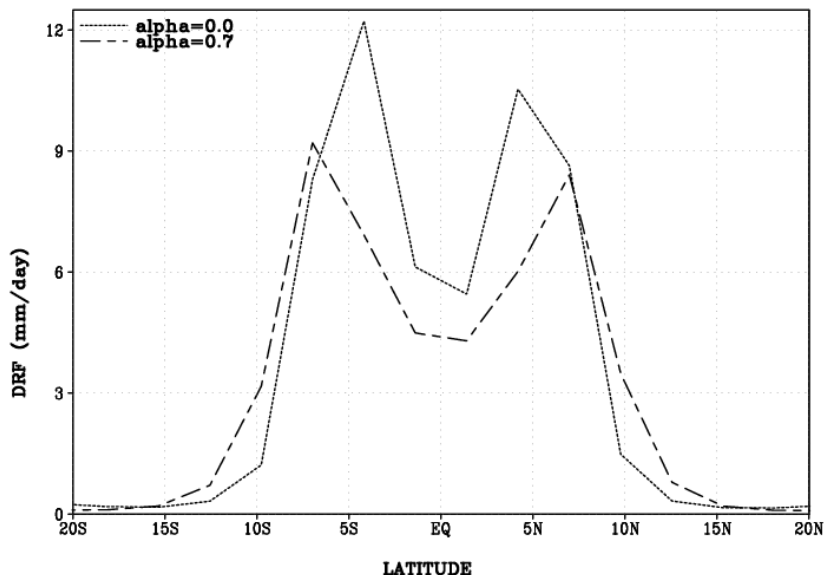

Fig. 1. Zonally averaged time mean deep convective rainfall (mm/day) with $\alpha=0$ and $\alpha=0.7$.

unanswered is whether or not the incorporation of downdrafts into a cumulus scheme results in a more stable atmosphere. Although Zhang has shown this in an SCM, it is necessary to examine this in a GCM, given the fact that feedback to the atmospheric circulation is absent in the former. This is the primary focus of this paper.

Section 2 discusses the model used and the experimental details. The impact of downdrafts on the simulation of the mean atmospheric stability is addressed in Sect. 3. In Sect. 4 we summarize the important results obtained from this study.

\section{Description of the model and experiments}

A detailed model description of CAM3 is given by Collins et al. (2004). Its main components are summarized as follows. The model uses a vertical coordinate, which is pure sigma near the surface, has a hybrid sigma-pressure in the middle troposphere and a pure pressure coordinate in the upper troposphere and above. There are 26 levels in the vertical. The semi-Lagrangian dynamical core with T42 truncation is used. Deep convection is parameterized by the Zhang and McFarlane (1995) cumulus scheme (ZM). Shallow convection is parameterized by using the Hack (1994) scheme (HK) and the prognostic cloud water parameterization scheme (RKZ) of Rasch and Kristjansson (1998), updated by Zhang et al. (2003) is used for the large-scale precipitation parameterization. The variables analyzed for the current work are deep convective rainfall, CAPE, rate of CAPE consumption, cloud base mass flux, updraft mass flux, rainfall production, sub-grid scale evaporation in the downdrafts, grid scale evaporation, entrainment into the updrafts, detrainment from the updrafts, relative humidity, temperature and specific humidity of the atmosphere.

The Sea Surface Temperature (SST) prescribed in the model is a continuous and smooth zonally symmetric func-

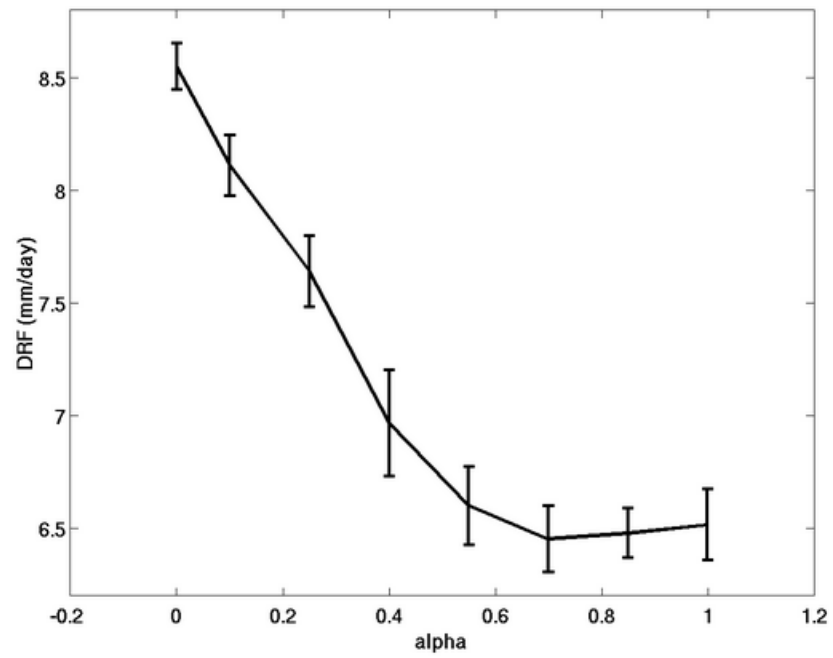

Fig. 2. Area averaged ( 0 to 360 and $7^{\circ} \mathrm{S}$ to $7^{\circ} \mathrm{N}$ ), time mean deep convective rainfall versus $\alpha$.

tion with the maximum at the equator. The SSTs are assumed to be invariant with longitude and their variation with latitude is the same as the control SST prescribed in accordance with the Neale and Hoskins protocol (Neale and Hoskins, 2000a) as follows:

$T_{S}(\lambda, \Phi)=\begin{array}{ll}27\left[1-\sin ^{2} \frac{(3 \Phi)}{2}\right]^{\circ} \mathrm{C}: \frac{-\pi}{3}<\Phi<\frac{\pi}{3} \\ 0^{\circ} \mathrm{C} \quad: \text { Otherwise }\end{array}$

where $\Phi$ is the latitude in radians, and $\lambda$ is the longitude. The maximum SST is $27^{\circ} \mathrm{C}$. SST retains a constant value of $0^{\circ} \mathrm{C}$ poleward of both $60^{\circ} \mathrm{S}$ and $60^{\circ} \mathrm{N}$. Sea-ice formation is neglected in the model. The solar forcing is set for equinoctial conditions (21 March) remaining symmetric about the equator but diurnal cycle is retained in the radiative forcing. The initial conditions for all the simulations are the same and are from a previous aqua-planet simulation, wherein the model was integrated for a period of 5 years. For all the simulations, the model was run for 18 months, the first six months of which were not considered due to spin up effects, and the last 12 months of data were considered for the analysis. As mentioned previously, we have followed the protocol of Neale and Hoskins (2000a) for our experiment. According to the protocol, adjustment to a change in the underlying SST occurs within the first 30-60 days of model integration. Hence, a 6-month period for spin up and a 12-month period for analysis is long enough. In order to verify whether 18 months of integration time is sufficient, we carried out two sets of integrations for a period of 4 years. It was seen that, although there exists a variability beyond 18 months, it is very small compared to the differences between the individual simulations. Hence, 18 months was chosen as the period for integration in all our experiments discussed in this paper. The monthly mean outputs were analyzed for all investigations in this work. 


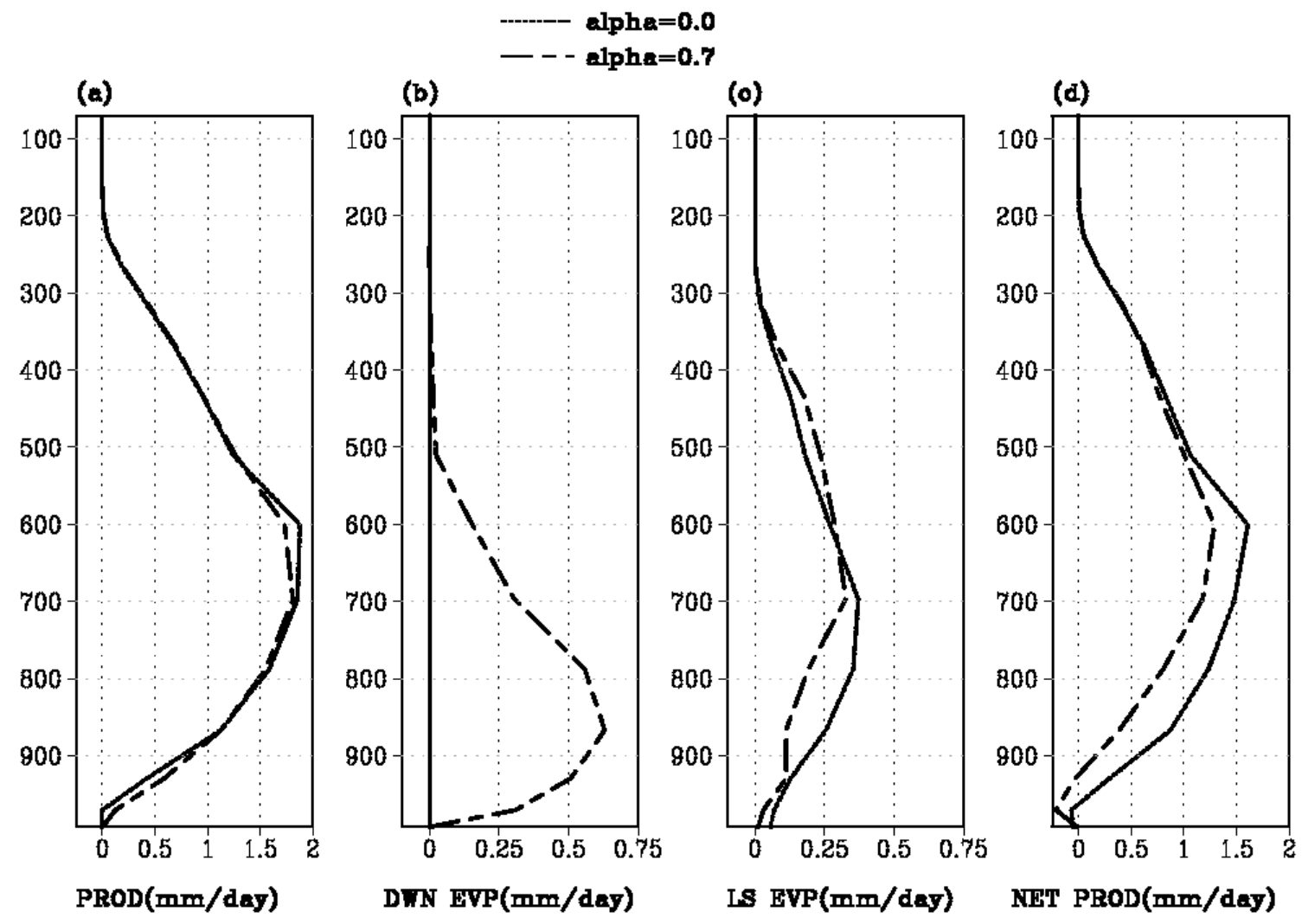

Fig. 3. Vertical profile of the area averaged ( 0 to 360 and $7^{\circ} \mathrm{S}$ to $7^{\circ} \mathrm{N}$ ), time mean, quantities for $\alpha=0$ and $\alpha=0.7$ (a) deep convective precipitation production rate ( $\mathrm{mm} /$ day), (b) evaporation of the deep convective precipitation inside the downdrafts (mm/day), (c) grid scale evaporation of the deep convective precipitation ( $\mathrm{mm} /$ day), (d) net deep convective precipitation production rate ( $\mathrm{mm} / \mathrm{day}$ ).

To address the questions posed in the Introduction, a series of experiments have been performed with different downdraft strengths in the cumulus scheme. The intensity of the downdrafts was varied by changing the value of the parameter $\alpha$ in the range of 0 to 1 , in the following downdraft formulation (Zhang and McFarlane, 1995), where the default value is 0.1 :

$M_{d}(Z)=\left(\left(-\alpha \times M_{b}\right) \times\left(\exp \left(\lambda_{m} \times\left(Z_{d}-Z\right)\right)-1\right)\right) /\left(\lambda_{m} \times\left(Z_{d}-Z\right)\right)$,

where $M_{d}$ is the downdraft mass flux at any height $Z, \alpha$ is the proportionality factor, $M_{b}$ is the cloud base mass flux, $\lambda_{m}$ is the maximum downdraft entrainment rate, $Z_{d}$ is the height of initiation of downdraft and $Z$ is the height.

\section{Impact of downdraft on deep convective rainfall}

Changes in the cumulus parameterization impacts computation of Deep Convective Rainfall (DRF). To locate the region of maximum impact we analyze the latitudinal variation of zonally averaged DRF (Fig. 1). It is seen from Fig. 1 that the region of significant impact is within $7^{\circ} \mathrm{S}$ to $7^{\circ} \mathrm{N}$ (deep tropics). Hence, analysis was focused only on the abovementioned region. The area average $\left(0\right.$ to 360 and $7^{\circ} \mathrm{S}$ to $\left.7^{\circ} \mathrm{N}\right)$ time mean (12 months) value of the total rainfall was found to be $8.9 \mathrm{~mm} /$ day when there were no downdrafts, whereas with the inclusion of the downdrafts the corresponding value is $7.4 \mathrm{~mm} /$ day. With the deep convective rainfall being the major component of the total rainfall, we further concentrate on this component alone. Figure 2 shows that the amount of deep convective rainfall, in the above-mentioned region is decreasing with an increase in the intensity of the downdrafts. The above figure shows that until $\alpha$ is 0.7 , the impact is monotonic, but after this, rainfall is largely insensitive to further changes in downdraft intensity. The default value of $\alpha$ used in CAM3.0 is 0.1. To understand the underlying mechanism relating DRF with downdraft intensity, the two extreme cases have been chosen for further analysis, viz. $\alpha=0$ and $\alpha=0.7$. The zonally averaged DRF was analyzed for both the simulations and it was seen that with the inclusion of downdrafts, there was a reduction in the zonally averaged deep convective rainfall over the deep tropics. This result is in agreement with that found by Sud and Walker (1993). The reduction in the zonally averaged value of deep convective rainfall in the presence of downdrafts in the cumulus scheme 

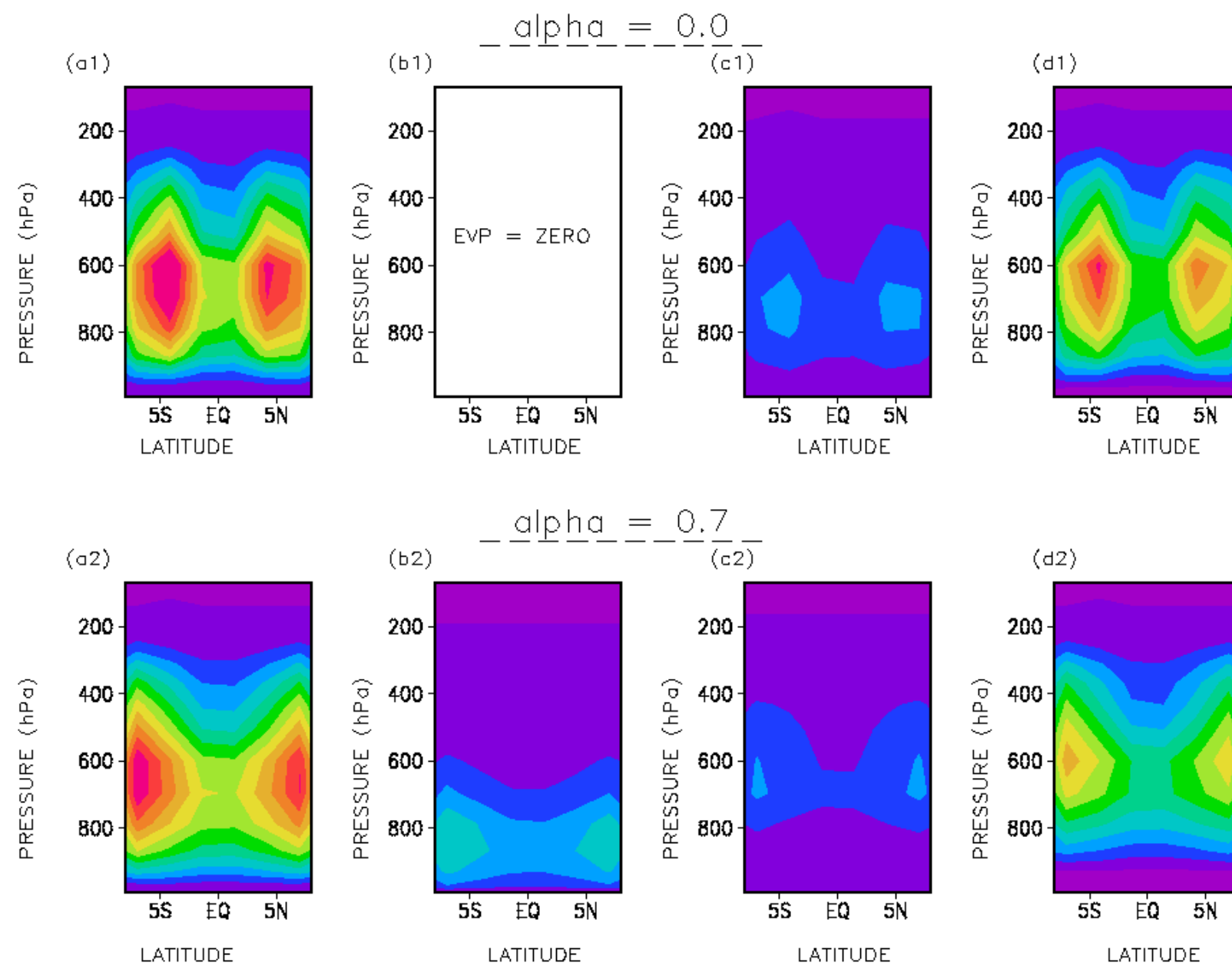

$=-\frac{0.7}{(\mathrm{c} 2)}$
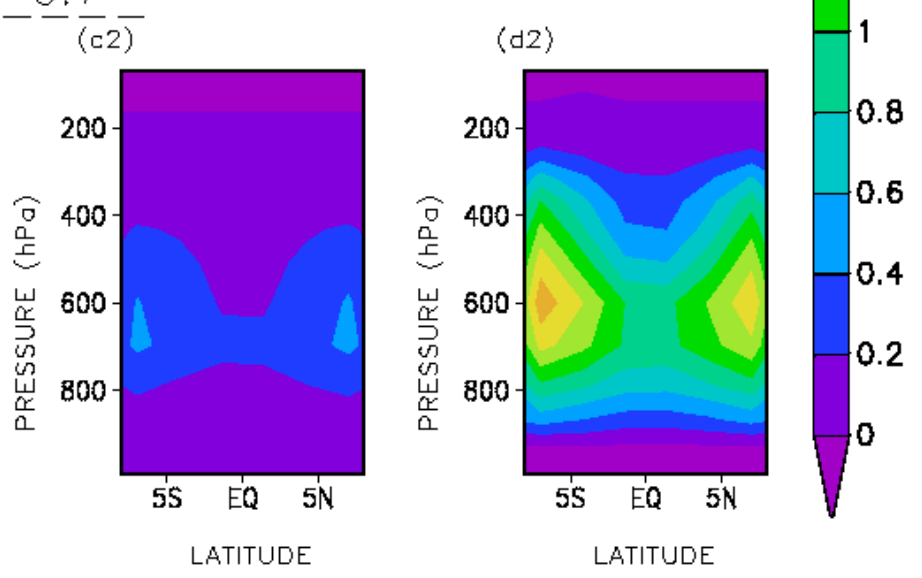

Fig. 4. Zonally averaged time mean quantities. Upper panel for $\alpha=0$ and lower panel for $\alpha=0.7$. (a1, a2) deep convective precipitation production rate (mm/day), (b1, b2) evaporation of the deep convective precipitation within the downdraft (mm/day), (c1, c2) grid scale evaporation of the deep convective precipitation $(\mathrm{mm} /$ day $),(\mathbf{d 1}, \mathbf{d 2})$ net deep convective precipitation production rate $(\mathrm{mm} / \mathrm{day})$.

could have been due either to a reduction in the production of precipitation or due to the evaporation of precipitation into the downdrafts to keep them saturated. We find that, although the production of precipitation is nearly the same in both cases (Fig. 3a), it is the evaporation of precipitation within the downdrafts (Fig. 3b) which leads to a reduction in the net precipitation rates (Fig. 3c) and hence the amount of deep convective rainfall (Fig. 2). This is further confirmed from Fig. 4 which shows the latitudinal variation of precipitation production, sub-grid scale evaporation of precipitation within the downdrafts, grid-scale evaporation of precipitation and the net precipitation production. We note that the maximum effect of the downdraft evaporation is in the lower troposphere. Grid scale evaporation of precipitating rainfall is found to be largely the same but net DRF rate reduces.

Another intriguing feature of the above results was that there was almost no change in the deep convective precipitation production in the two simulations. To investigate these various terms involved in the formulation of precipitation, production in the ZM scheme was analyzed. The amount of rain water that will be formed out of the cloud water is formulated in the following way in the ZM scheme (Zhang and McFarlane, 1995):

$R=C_{0} \times M_{u} \times l$,

where $R$ is the rate of precipitation production $(\mathrm{kg} / \mathrm{kg} / \mathrm{s}), C_{0}$ is the deep convection precipitation production efficiency parameter $\left(3 \times 10^{-3} \mathrm{~m}^{-1}\right), M_{u}$ is the updraft mass flux, and $l$ is the cloud liquid water.

It can be seen from Fig. 5 that with the inclusion of convective downdrafts into the cumulus parameterization scheme there was a reduction in the updraft mass flux which is found to be larger at lower levels. To investigate further the reason behind the reduction in the upward mass flux, the cloud base mass flux was analyzed and it was found that the area averaged time mean value of the cloud base mass flux for the cases with $\alpha=0.0$ and $\alpha=0.7$ were found to be $96.2 \mathrm{hPa} / \mathrm{day}$ and $74.2 \mathrm{hPa} /$ day, respectively, which could be the reason for 

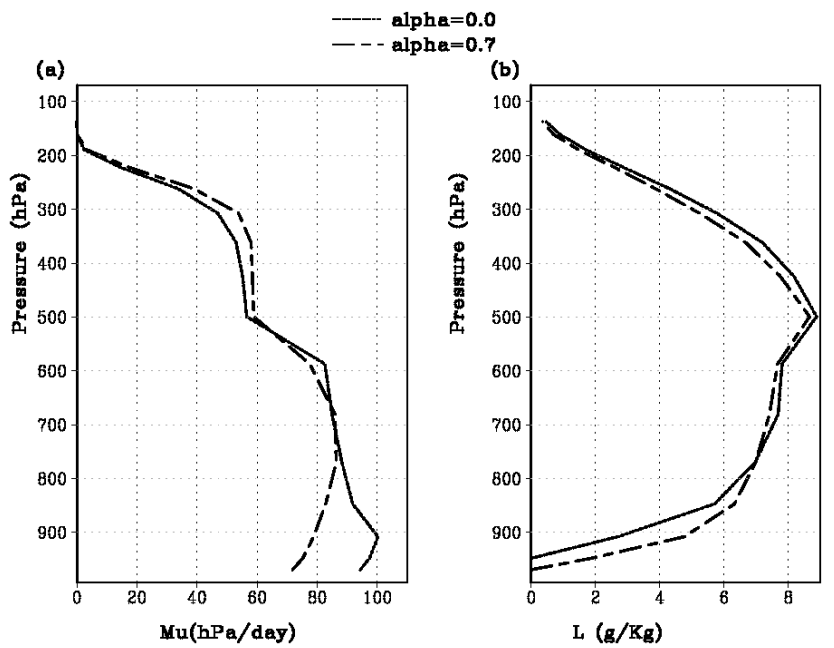

Fig. 5. Vertical profile of the area averaged $\left(0\right.$ to 360 and $7^{\circ} \mathrm{S}$ to $7^{\circ} \mathrm{N}$ ), time mean, quantities for $\alpha=0$ and $\alpha=0.7$ (a) Updraft mass flux (hPa/day), (b) cloud liquid water $(\mathrm{g} / \mathrm{kg})$.

the reduction in the updraft mass flux in the lower levels. The differences in updraft mass flux (Fig. 6a), the entrainment and detrainment profiles (Fig. 7b, c) were analyzed and found to be consistent. The lower updraft mass flux was due to the combined effect of cloud base mass flux, detrainment and entrainment.

We next analyzed the reason behind the reduction in the cloud base mass flux with the incorporation of downdrafts. The cloud base mass flux is formulated in the following way in the ZM scheme, based on the closure condition that, with CAPE removed at an exponential rate by convection with a characteristic adjustment time scale $\tau$ :

$M_{b}=A /(\tau \times F)$,

where $A$ is the excess CAPE above the specified threshold, $\tau$ is the adjustment time scale and $F$ is the rate of CAPE consumption per unit cloud base mass flux. In the above formulation, CAPE is the convective available potential energy at the instant when the ZM scheme is triggered; the cape threshold has a constant value of $70 \mathrm{~J} / \mathrm{kg}$ and the adjustment time scale has a constant value of $3600 \mathrm{~s}$.

Since the adjustment time scale and the cape threshold are the same for both the simulations, the reason behind the differences in the cloud base mass flux could be either due to the CAPE or due to the rate of CAPE consumption per unit cloud base mass flux.

From the analysis of CAPE (Fig. 7), it was seen that the inclusion of downdrafts in the ZM scheme actually leads to an increase in the CAPE which is a measure of atmospheric instability. This result is contrary to what ZM (1995) had concluded in their SCM study. It was stated in ZM (1995) that strong downdrafts provide a powerful mechanism in stabilizing the atmospheric column. In a SCM, feedback from the
Table 1. Area averaged value of CINE (J/kg) for $\alpha=0.0$ and $\alpha=0.7$.

\begin{tabular}{cccc}
\hline $\begin{array}{c}\text { Value } \\
\text { of } \alpha\end{array}$ & $\begin{array}{c}0-360, \\
7^{\circ} \mathrm{S} \text { to } 7^{\circ} \mathrm{N}\end{array}$ & $\begin{array}{c}0-360, \\
15^{\circ} \mathrm{S} \text { to } 15^{\circ} \mathrm{N}\end{array}$ & $\begin{array}{c}0-360, \\
30^{\circ} \mathrm{S} \text { to } 30^{\circ} \mathrm{N}\end{array}$ \\
\hline 0.0 & 3.1 & 1.7 & 0.7 \\
0.7 & 5.3 & 6.5 & 3.4 \\
\hline
\end{tabular}

cumulus scale to the large-scale environment is absent and the circulation fields are not related to changes in convective heating. On the contrary in a GCM, the circulation and heating fields have to be consistent and the circulation becomes modified by the convective heating. Other differences, such as those played by the boundary layer parameterization in handling moisture transported by the downdrafts, may also play a role.

An increase in CAPE (Fig. 7) should favor an increase in $M_{b}$, but we find that on the contrary, the cloud base mass flux is actually lower. The area averaged time mean value for the cloud base mass flux is found to be $96.2 \mathrm{hPa} / \mathrm{day}$, without downdrafts, whereas it is $74.2 \mathrm{hPa} /$ day with downdrafts. This is a clear indication of the fact that the increase in $F$ is such that it more than compensates for the increase in $M_{b}$ due to the increase in CAPE and leads to a final reduction in $M_{b}$, which could be noticed from Fig. 7c. The increase in $F$ with the inclusion of downdrafts can be partly attributed to the increase in the convection inhibition energy (CINE). The area averaged time mean value for CINE is $3.1 \mathrm{~J} / \mathrm{kg}$, without downdrafts, whereas it is $5.3 \mathrm{~J} / \mathrm{kg}$ with downdrafts for the deep tropics $\left(7^{\circ} \mathrm{N}\right.$ to $\left.7^{\circ} \mathrm{S}\right)$, as can be seen from Table 1 , where the area averaged time mean CINE values are consistently higher throughout the tropics, and hence lead to an increased consumption of CAPE for convection to occur.

We further investigate the effect of convective scale downdrafts on large-scale temperature and moisture profiles. We find that (Fig. 8) there is drying near the surface and above $600 \mathrm{hPa}$. Lower and mid-troposphere (Fig. 8a) are more moist with the inclusion of downdrafts. These results are consistent with those of Moorthi and Suarez (1999). The large-scale temperature profile shows cooling throughout the troposphere with the inclusion of downdrafts. The relative humidity, on the other hand, being controlled by the net effect of cooling, and drying is found to increase up to $600 \mathrm{hPa}$, with the inclusion of downdrafts.

Incorporation of the downdraft leads to an increase in the specific humidity in the lower troposphere, which is shown in Fig. 9a1 and a2. In contrast, atmospheric temperature is reduced almost throughout the atmosphere, starting from the surface up to $100 \mathrm{hPa}$, which can be noticed from Fig. 9b1 and b2. Moreover, the temperature response is neither occurring locally in the vertical, like the specific humidity, nor having much gradient in the vertical; instead, a largely uniform cooling occurs almost throughout the lower and mid 
(a)

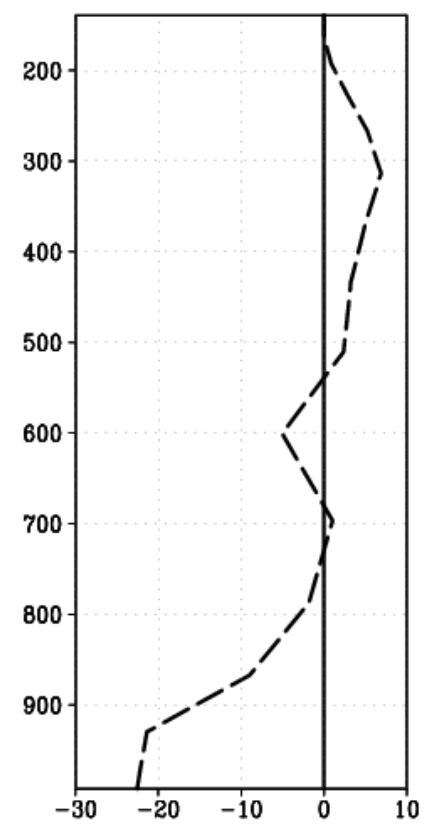

(b)

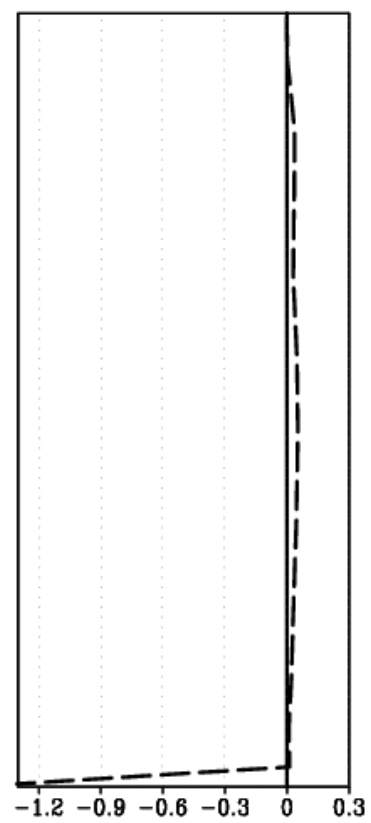

(c)

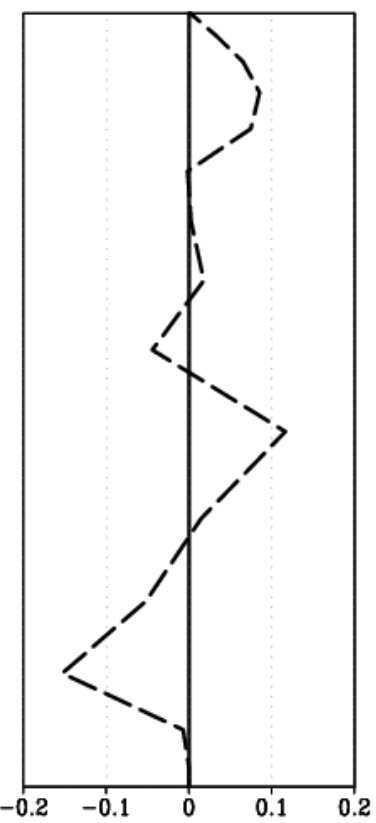

Fig. 6. Vertical profile of the area averaged ( 0 to 360 and $7^{\circ} \mathrm{S}$ to $\left.7^{\circ} \mathrm{N}\right)$ time mean differences $(\alpha=0.7-\alpha=0.0)$. (a) Updraft mass flux (hPa/day) (b) Entrainment (c) Detrainment.

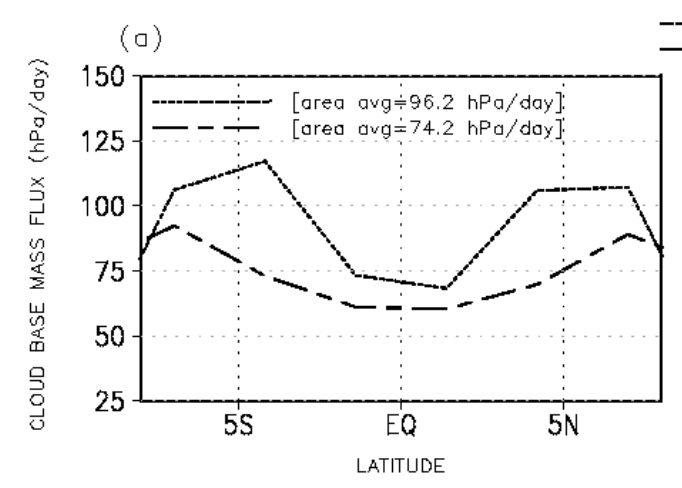

--C---- alpha $=0.0$

(b)
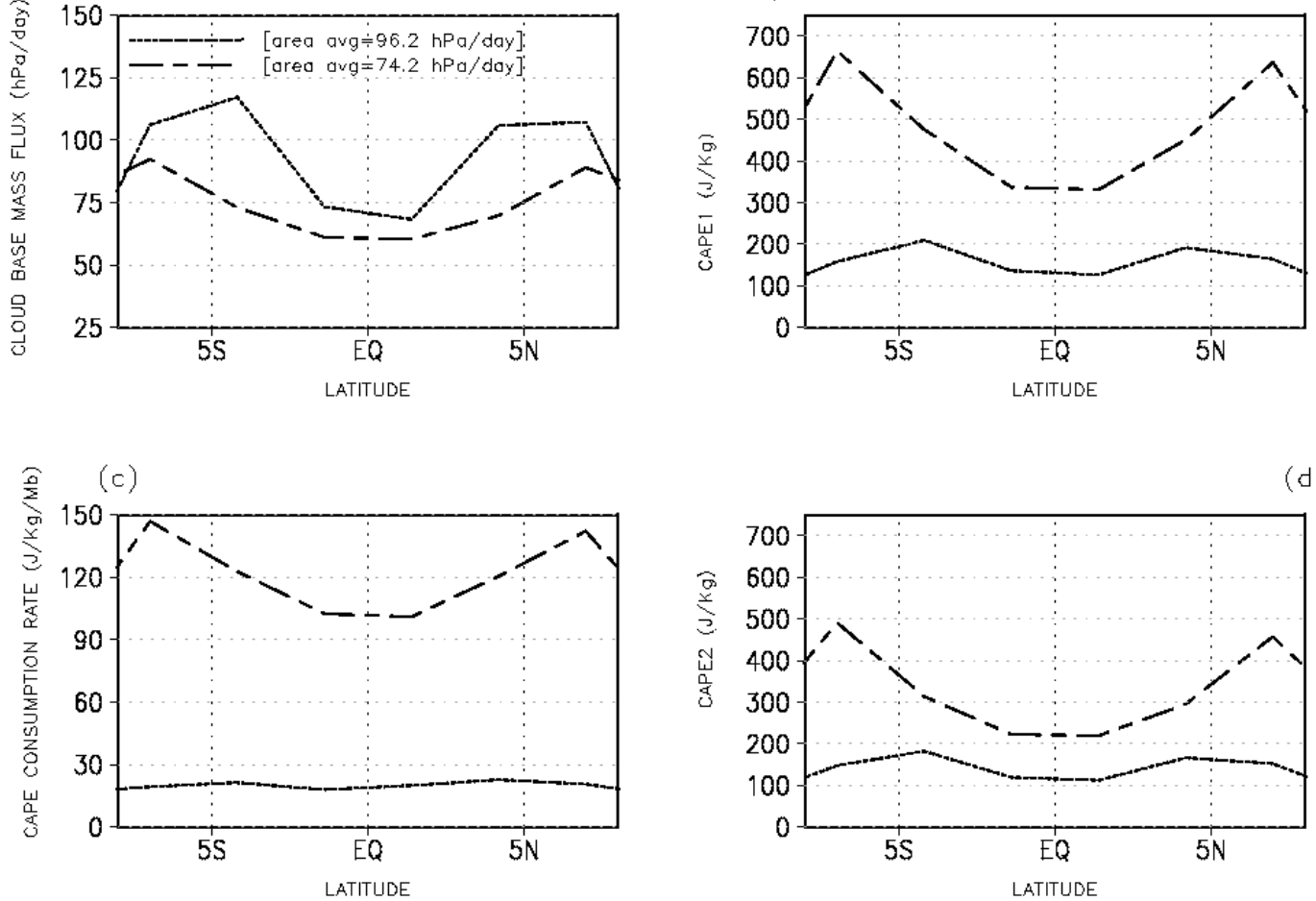

(d)

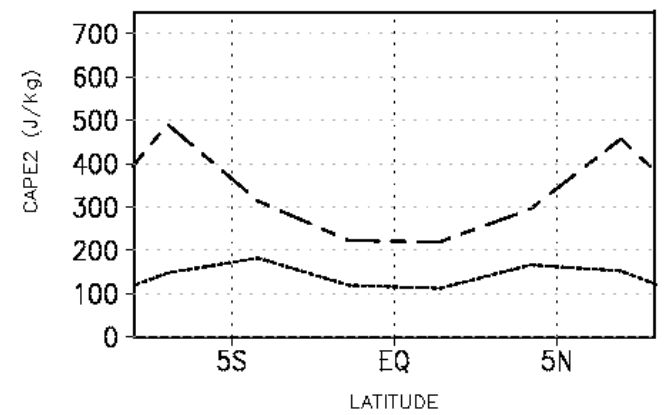

Fig. 7. Zonally averaged time mean quantities for $\alpha=0$ and $\alpha=0.7$. (a) cloud base mass flux (hPa/day), (b) CAPE just before the deep convective scheme is invoked $(\mathrm{J} / \mathrm{kg})$, (c) CAPE consumption rate $\left(\mathrm{J} / \mathrm{kg} / \mathrm{M}_{b}\right)$, (d) CAPE just after computations in deep convective scheme $(\mathrm{J} / \mathrm{Kg})$. 


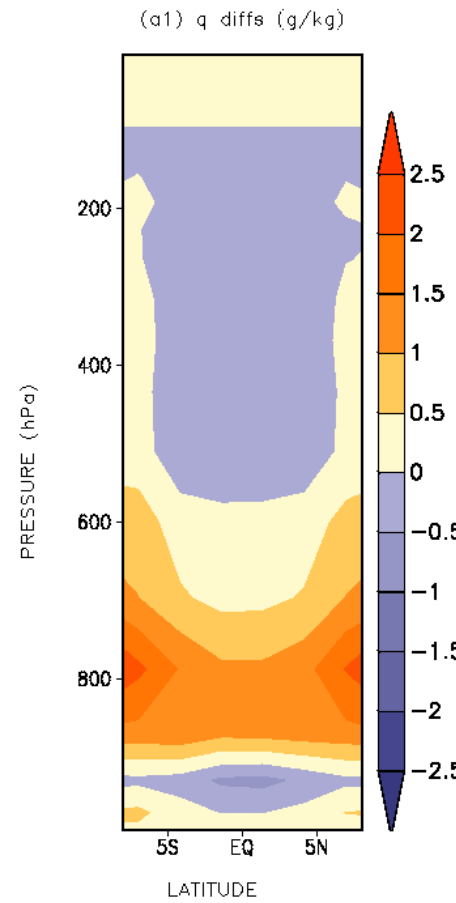

(b1) $T$ diffs (K)
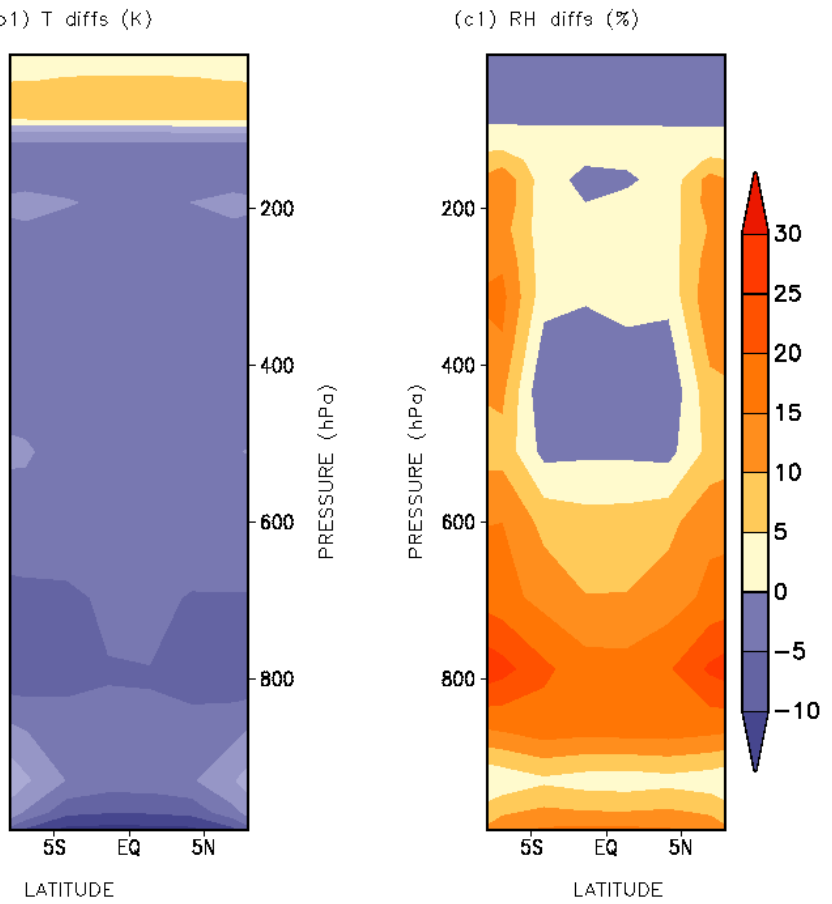

Fig. 8. Vertical profile of the difference between the zonally averaged ( 0 to 360), time mean, quantities between $\alpha=0.7$ and $\alpha=0.0$. (a) specific humidity difference $(\mathrm{g} / \mathrm{kg})$, (b) atmospheric temperature difference $(\mathrm{K})$ (c) $\mathrm{RH}$ difference $(\%)$.
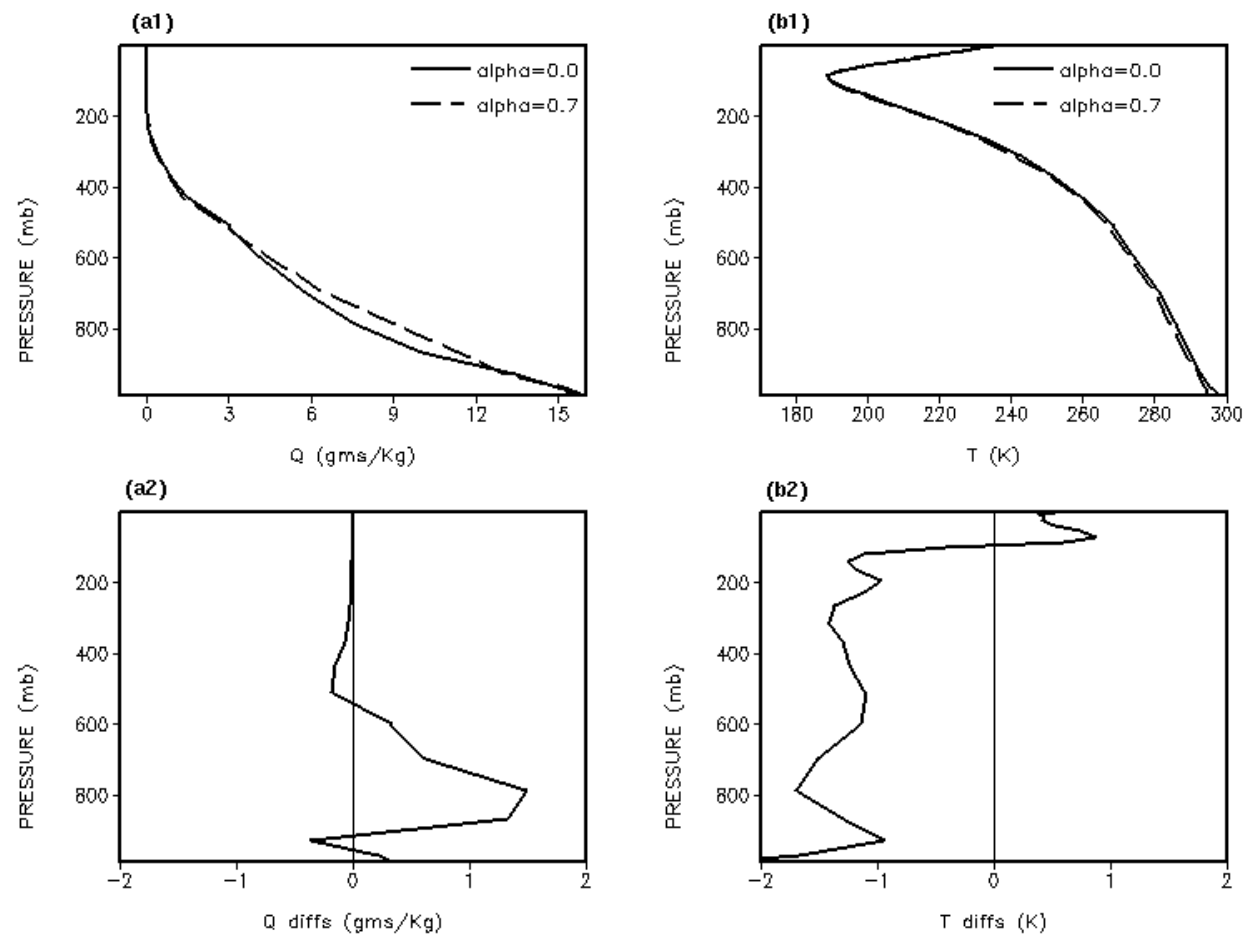

Fig. 9. Vertical profile of the the area averaged ( 0 to 360 and $7^{\circ} \mathrm{S}$ to $7^{\circ} \mathrm{N}$ ), time mean, quantities, (a1) specific humidity for $\alpha=0.7$ and $\alpha=0.0$. (g/kg), (b1) atmospheric temperature for $\alpha=0.7$ and $\alpha=0.0(\mathrm{~K})$. (a2) specific humidity difference between $\alpha=0.7 \mathrm{and} \alpha=0.0$. ( $\mathrm{g} / \mathrm{kg}$ ), (b2) atmospheric temperature difference between $\alpha=0.7$ and $\alpha=0.0(\mathrm{~K})$. 

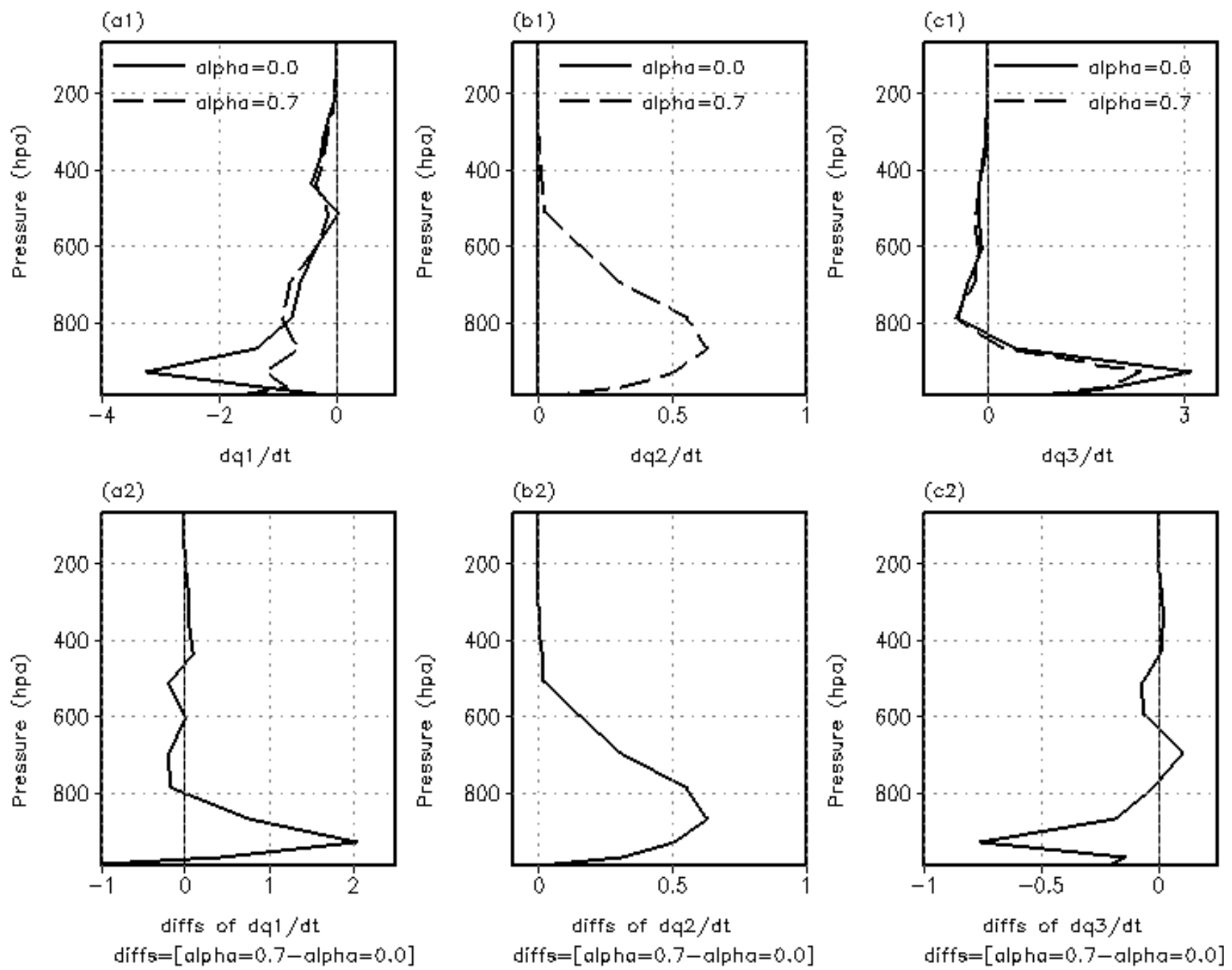

Fig. 10. Vertical profile of the the area averaged ( 0 to 360 and $7^{\circ} \mathrm{S}$ to $\left.7^{\circ} \mathrm{N}\right)$, time mean, quantities, (a1) dq1/dt (specific humidity tendency due to condensation) for $\alpha=0.7$ and $\alpha=0.0$. ( $\mathrm{kg} / \mathrm{m}^{2} / \mathrm{day}$ ), (b1) dq2 $/ \mathrm{dt}$ (specific humidity tendency due to evaporation in the downdraft) for $\alpha=0.7$ and $\alpha=0.0\left(\mathrm{~kg} / \mathrm{m}^{2} / \mathrm{day}\right)$, (c1) dq3 $/ \mathrm{dt}$ (specific humidity tendency due to lateral moisture advection into the region) for $\alpha=0.7$ and $\alpha=0.0$ ( $\mathrm{kg} / \mathrm{m}^{2} /$ day), (a2) dq1/dt difference between $\alpha=0.7$ and $\alpha=0.0\left(\mathrm{~kg} / \mathrm{m}^{2} / \mathrm{day}\right)$, (b2) $\mathrm{dq} 2 / \mathrm{dt}$ difference between $\alpha=0.7$ and $\alpha=0.0\left(\mathrm{~kg} / \mathrm{m}^{2} / \mathrm{day}\right)$, (c2) dq3/dt difference between $\alpha=0.7$ and $\alpha=0.0\left(\mathrm{~kg} / \mathrm{m}^{2} /\right.$ day).

troposphere. The responses of specific humidity and temperature illustrated in the above-mentioned figures, indicate that the reason behind the increase of CAPE is due to the increase of specific humidity in the lower troposphere.

The above-mentioned response of specific humidity can be attributed to the combined effect of the following three factors: (1) condensation in the lower troposphere, (2) reevaporation of moisture in the downdraft and, (3) lateral moisture advection into this region in the lower troposphere. The three factors stated above could be noticed in Fig. 10. The specific humidity tendency due to condensation is less negative in the presence of downdrafts, which leads to an increase in the specific humidity in the lower troposphere, as can be seen from Fig. 10a1. The specific humidity tendency due to evaporation of rainfall is zero in the absence of downdrafts, whereas it is positive in the presence of downdrafts and hence leads to an increase in specific humidity in the lower and mid troposphere, as can be seen from Fig. 10a2. The specific humidity tendency due to lateral moisture transport is less in the presence of downdrafts, which explains the low specific humidity in the presence of downdrafts around $925 \mathrm{hPa}$ in Fig. 9a2.

As there were significant changes in the temperature and moisture fields it was expected that there would also be an associated change in the cloud field and the net cloud radiative forcing. We further analyzed the changes in the cloud fraction for the low, middle and high clouds and the associated cloud radiative forcing, both in the long wave and the 

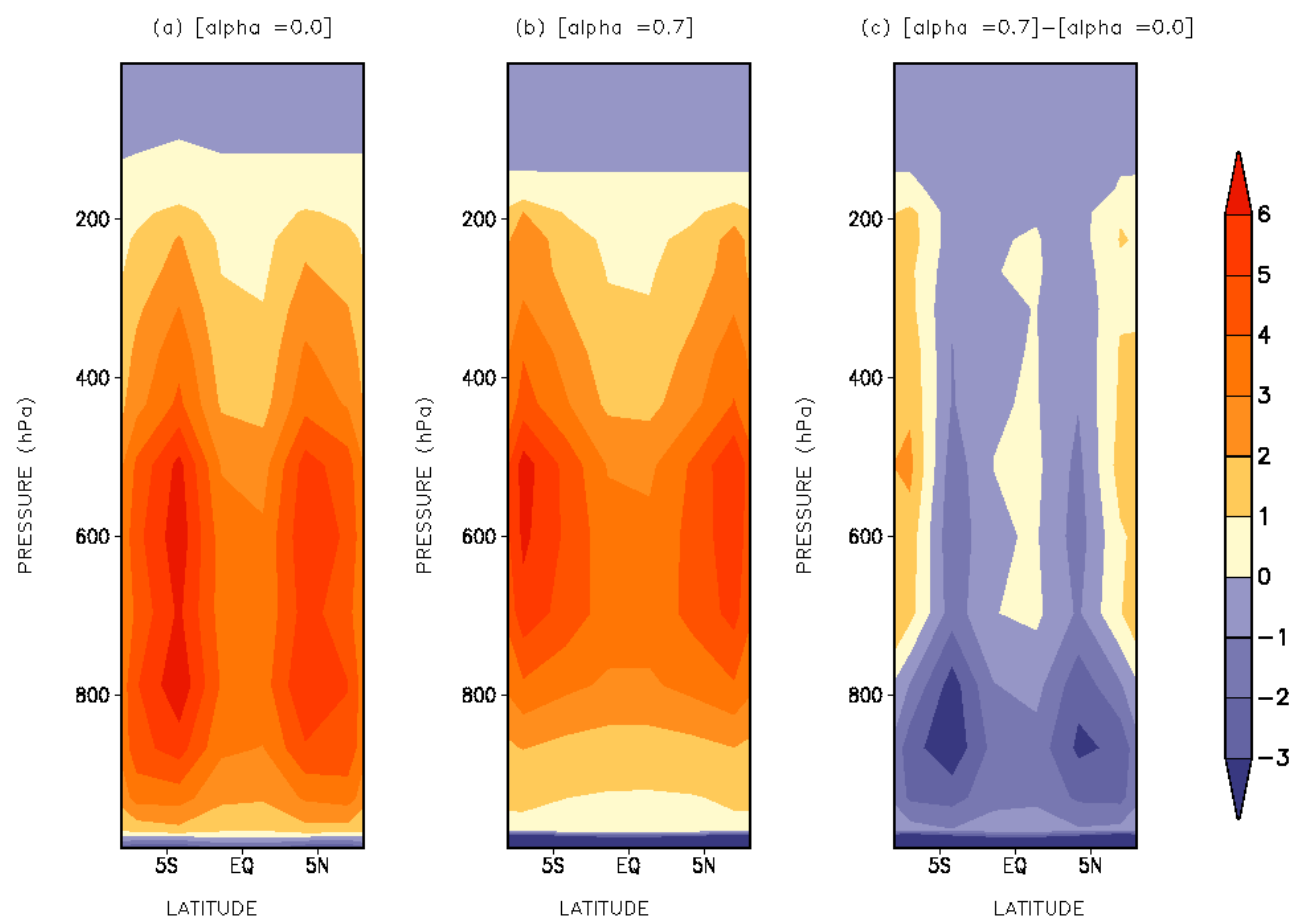

Fig. 11. Zonally averaged time mean temperature tendency due to ZM moist convection scheme (K/day) (a) $\alpha=0.0$, (b) $\alpha=0.7$, and (c) $(\alpha=0.7)-(\alpha=0.0)$.
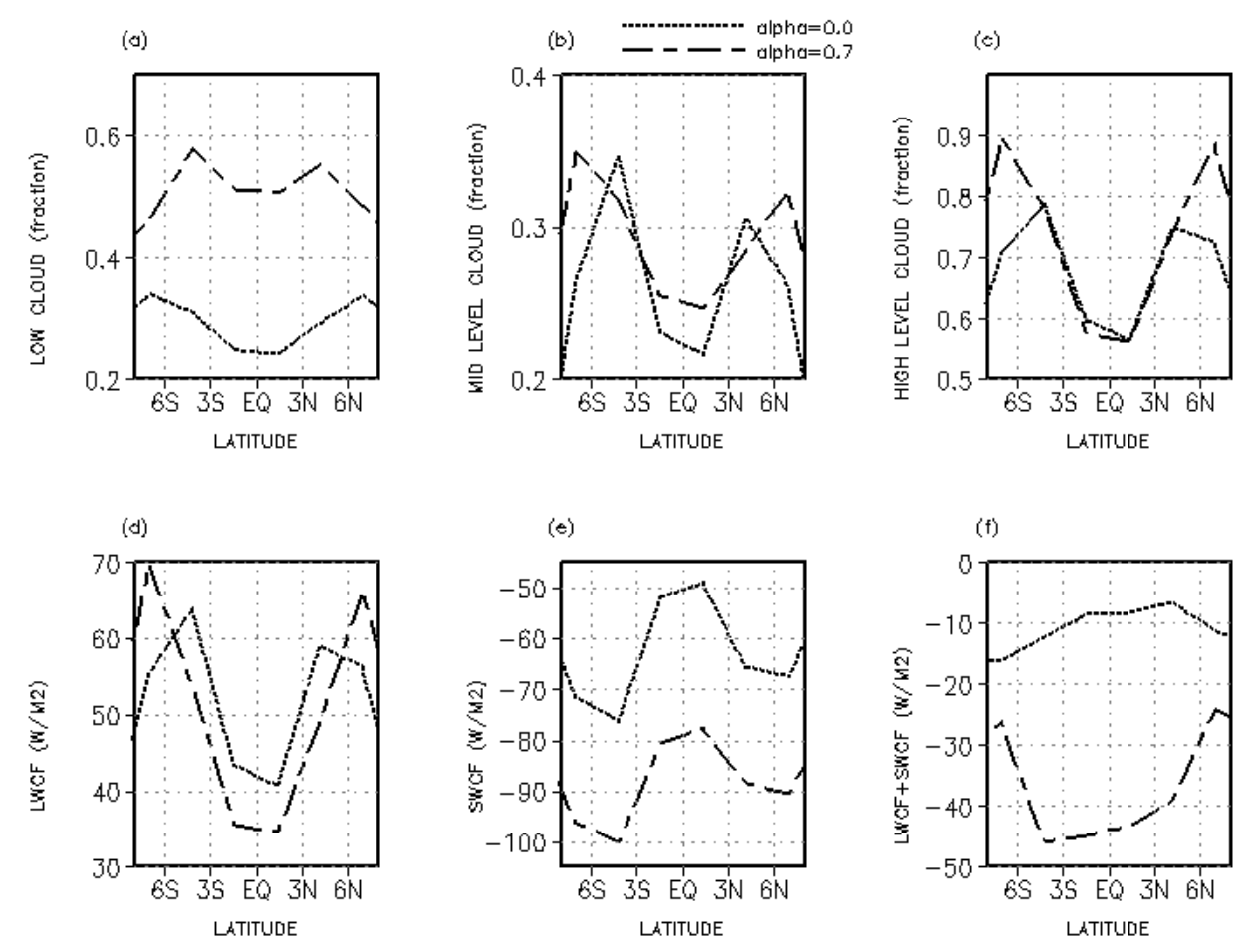

Fig. 12. Zonally averaged time mean quantities for $\alpha=0$ and $\alpha=0.7$. (a) low cloud (fraction), (b) mid level cloud (fraction), (c) high level cloud (fraction), (d) longwave cloud radiative forcing $\left(\mathrm{W} / \mathrm{m}^{2}\right)$, (e) shortwave cloud radiative forcing $\left(\mathrm{W} / \mathrm{m}^{2}\right)$, (f) net (long wave +short wave) cloud radiative forcing $\left(\mathrm{W} / \mathrm{m}^{2}\right)$. 

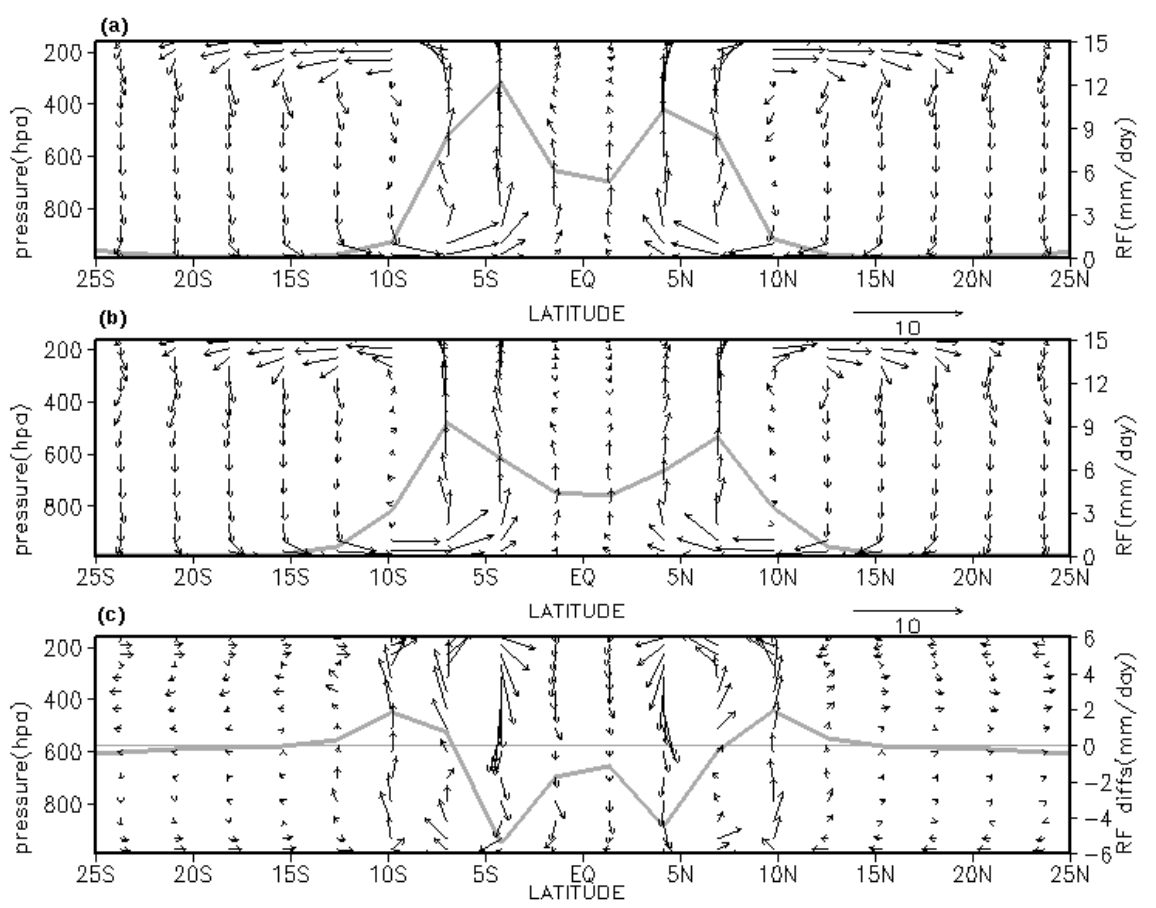

Fig. 13. Zonally averaged time mean meridional circulation (vector arrows: $v ; 50 \times w$ ) and rainfall (grey line) for (a) $\alpha=0.0$, and (b) $\alpha=0.7$ and (c) $(\alpha=0.7)-(\alpha=0.0)$.

short wave. The reduction in the large-scale temperature can be largely explained by the combined effect due to the reduction in the temperature tendency, due to the cumulus parameterization, as can be seen from Fig. 11 and the large increase in the low cloud fraction accompanied by an increase in the shortwave cloud forcing, as can be seen from Fig. 12. The effect on long wave cloud forcing is much smaller and the change in total cloud radiative forcing is dominated by short wave cloud forcing.

The other characteristic change due to the incorporation of downdrafts is the modification of the atmospheric circulation. From Fig. 13 it can be seen that the rainfall over the equatorial region becomes lower and the Inter Tropical Convergence Zone (ITCZ) becomes broader with the inclusion of downdrafts. A comparison of the circulation depicts that, with the inclusion of downdrafts, the upward equatorial limb of the Hadley cell becomes weaker and wider (in consistence with the ITCZ).

\section{Summary and conclusions}

The impact of downdrafts on the simulated mean atmospheric state is analyzed. It is noticed that the instability of the atmosphere measured in terms of CAPE increases with the increase in intensity of downdrafts. Thus, in the current work it is shown that the formulation of downdrafts in the current version of the ZM scheme used in the NCAR CAM3.0 is, in fact, making the atmosphere more unstable, which is seen from the increase in CAPE with the inclusion of convective downdrafts into the cumulus scheme, as compared to the case when there are no downdrafts. This result is contrary to what was found by Zhang and McFarlane (1995) in their study using a single column model.

The current work shows that there is an increase in the rate of consumption of CAPE with the inclusion of downdrafts in the cumulus scheme, which is in agreement with one of the results of ZM (1995) and Molinari and Corsetti (1985). Even though the CAPE consumption rate is higher, the model climate is found to shift to a regime with a greater amount of CAPE and hence, instability in the atmosphere.

It is seen that there is a reduction in the deep convective rainfall with the inclusion of downdrafts in the ZM scheme, which is consistent with the earlier studies on downdrafts (e.g. Sud and Walker, 1993). This reduction in the deep convective rainfall is shown to be primarily due to the evaporation of rainfall within the downdrafts. It is further shown that although the production of precipitation is found to be the same in both cases, there is a decrease in the updraft mass flux in the presence of downdrafts. The updraft mass flux is found to reduce due to the lowering of the cloud base mass flux, which, in turn, is found to be less, due to the compensating effect of the rate of consumption of CAPE on the increase of CAPE.

This discrepancy in the model against the general belief that downdrafts stabilize the atmosphere, could be either due to the closure employed in the current version of the $\mathrm{ZM}$ 
scheme or other assumptions made in the formulation of downdrafts. Since CAPE is largely determined by the boundary layer temperature and moisture (Zhang, 2003a; Donner and Phillips, 2003) and it is seen that convective downdrafts significantly modify the thermodynamic properties of the air in the planetary boundary layer, certain assumptions, such as keeping the downdrafts in a saturated state, made in the current version of the $\mathrm{ZM}$ scheme might have led to higher values of CAPE simulated with the inclusion of downdrafts.

It is also observed in the current work that drying occurs near the surface and above $600 \mathrm{hPa}$, whereas moistening occurs in the lower and mid troposphere. The large-scale temperature profile shows cooling throughout the troposphere with the inclusion of downdrafts. The relative humidity, on the other hand, being controlled by the net effect of cooling and drying, is found to increase up to $600 \mathrm{hPa}$, with the inclusion of downdrafts.

The specific humidity near the surface is less in the presence of downdrafts due to lower lateral advection of moisture, whereas it is found to be more in the lower and mid troposphere due to the evaporation of falling precipitation. The reduction in large-scale temperature can be largely explained by the combined effect due to a reduction in temperature tendency because of the cumulus parameterization and large increase in the low cloud fraction, accompanied by an increase in the shortwave cloud forcing.

Another important finding is the broadening of the ITCZ in the presence of downdrafts which can be attributed to the change in the structure of the Hadley cell. It is observed that the upward equatorial limb of the Hadley cell becomes weaker and wider in the presence of downdrafts.

These conclusions are based on simulations in an aquaplanet framework. It is also necessary to study the effect of convective downdrafts in a model with realistic land-ocean configurations. Response of the model to the inclusion of downdrafts could differ in the presence of land-ocean feedbacks.

Acknowledgements. V. Venugopal is thankfully acknowledged for all the encouragement during the course of this work. We thank SERC-IISc, for the computational support. This work is partially supported by India Meteorological Department. We would also like to thank the two anonymous reviewers for their constructive comments.

Topical Editor F. D'Andrea thanks two anonymous referees for their help in evaluating this paper.

\section{References}

Collins, W. D., Rasch, P. J., Boville, B. A., et al.: Description of the NCAR Community Atmosphere Model (CAM 3.0). NCAR Technical Note, NCAR/TN-464+STR, 226 pp, 2004.

Donner, L. J. and Phillips, V. T.: Boundary layer control on convective available potential energy: Implications for cumulus parameterization, J. Geophys. Res., 108(D22), 4701, doi:10.1029/2003JD003773, 2003.

Molinari, J. and Corsetti, T.: Incorporation of cloud scale and mesoscale downdrafts into a cumulus parameterization: Results of One- and Three-Dimensional Integrations, Mon. Weather Rev., 113, 485-501, 1985.

Moorthi, S. and Suarez, M. J.: Documentation of Version 2 of Relaxed Arakawa-Schubert Cumulus Parameterization with Convective Downdrafts, NOAA Tech. Rep. NWS/NCEP 99-01, U.S. Department of Commerce, 44pp, 1999.

Neale, R. B. and Hoskins, B. J.: A standard test for AGCMs including their physical parameterizations: I: The Proposal, Atmos. Sci. Lett., 1, 101-107, 2000a.

Neale, R. B. and Hoskins, B. J.: A standard test for AGCMs including their physical parameterizations. II: results for the Met Office Model, Atmos. Sci. Lett., 1, 108-114, 2000b.

Rasch, P. J. and Kristjansson, J. E.: A comparison of the CCM3 model climate using diagnosed and predicted condensate parameterizations, J. Climate, 11, 1587-1614, 1998.

Sud, Y. C. and Walker, G. K.: A rain evaporation and downdraft parameterization to complement a cumulus updraft scheme and its evaluation using GATE data, Mon. Weather Rev., 121, 30193039, 1993.

Zhang, M., Lin, W., Bretherton, C. S., Hack, J. J., and Rasch, P. J.: A modified formulation of fractional stratiform condensation rate in NCAR community atmospheric model CAM2, J. Geophys. Res., 108(D1), 4035, doi:10.1029/2002JD002523, 2003.

Zhang, G. J. and McFarlane, N. A.: Sensitivity of climate simulations to the parameterization of cumulus convection in the Canadian Climate Centre General Circulation Model, Atmos.-Ocean, 33(3) 407-446, 1995.

Zhang, G. J.: Convective quasi-equilibrium in mid-latitude continental environment and its effect on convective parameterization, J. Geophys. Res., 107(D14), 4220, doi:10.1029/2001JD001005, 2002.

Zhang, G. J.: Convective quasi-equilibrium in the tropical western Pacific: Comparison with midlatitude continental environment, J. Geophys. Res., 108(D19), 4592, doi:10.1029/2003JD003520, 2003.

Zhang, G. J. and Mu, M.: Effects of modifications to the ZhangMcFarlane convection parameterization on the simulation of the tropical precipitation in the National Center for Atmospheric Research Community Climate Model, version 3, J. Geophys. Res., 110, D09109, doi:10.1029/2004JD005617, 2005. 